\title{
Téoros
}

Revue de recherche en tourisme

\section{Tourisme, géosciences et enjeux de territoires}

Actualités du géotourisme

\section{Mélanie Duval et Christophe Gauchon}

Volume 29, numéro 2, 2010

Géotourisme

URI : https://id.erudit.org/iderudit/1024865ar

DOI : https://doi.org/10.7202/1024865ar

Aller au sommaire du numéro

Éditeur(s)

Université du Québec à Montréal

ISSN

0712-8657 (imprimé)

1923-2705 (numérique)

Découvrir la revue

Citer ce document

Duval, M. \& Gauchon, C. (2010). Tourisme, géosciences et enjeux de territoires : actualités du géotourisme. Téoros, 29(2), 3-14.

https://doi.org/10.7202/1024865ar d'utilisation que vous pouvez consulter en ligne.

https://apropos.erudit.org/fr/usagers/politique-dutilisation/ 


\title{
Tourisme, géosciences et enjeux de territoires Actualités du géotourisme
}

\author{
Mélanie DUVAL \\ Chargée de recherche CNRS \\ Laboratoire Edytem, Université de Savoie \\ melanie.duval@univ-savoie.fr \\ Christophe GAUCHON \\ Maître de conférences \\ Université de Savoie \\ christophe.gauchon@univ-savoie.fr
}

Depuis les années 1990, le vocabulaire du tourisme s'est enrichi de déclinaisons nouvelles autour de l'écotourisme, du cybertourisme, du tourisme doux, du tourisme durable et/ ou responsable... Dans le même temps, une famille de mots nouveaux apparaissaient, articulés sur le préfixe «géo-» : géopatrimoine, géotope, géodiversité, géoparc, géosymbole, géodiscours, géoproduit, géovisualisation et bien sûr géotourisme. Certains sont restés confidentiels et ne circulent encore que dans des cercles restreints. D'autres ont acquis une audience plus large, parfois à travers des procédures d'agrément ou de labellisation. D'autres encore appartiennent au jargon des opérateurs, mais ils ont leur utilité et on les trouvera employés dans le présent volume consacré au géotourisme. Il est possible que la grande audience des thèses de J. Lovelock et la popularisation du système Gaïa (quoiqu'on en pense sur le fond) aient contribué à ces innovations lexicales et à leur diffusion (Lovelock, 1986).

En lien avec les stratégies des acteurs, l'évolution de la demande, les innovations techniques et les logiques patrimoniales, les dynamiques touristiques évoluent, se réajustent, se transforment, jouant la carte du kaléidoscope. Pourl'essentiel, le géotourisme a été abordé par les géologues et géomorphologues dans des logiques d'inventaires (Fernanda de Lima et al., 2010), laissant peu de place à l'approche territoriale des enjeux touristiques et patrimoniaux. L'objectif de ce dossier thématique est très justement d'interroger la teneur géographique du géotourisme ainsi que les réalités qu'il recouvre : quels en sont les éléments de définition et les spécificités? En quoi le géotourisme se distingue-t-il de ces voisins «écotourisme» et «tourisme culturel»? S'agit-il de nouvelles pratiques touristiques? Dans quelle mesure cette nouvelle appellation fait-elle sens et permet-elle à la recherche en tourisme d'avancer?

Au-delà d'un questionnement théorique sur les contours de cette notion, il y a une réelle nécessité sociale de faire le point. En effet, à l'heure actuelle, de plus en plus d'acteurs mettent en avant le géotourisme dans divers projets de territoire, sans pour autant que celui-ci soit clairement défini en amont. Or, l'efficacité, la dimension opératoire d'une notion, passe par des efforts de clarification. S'il n'est pas question d'enfermer le géotourisme dans un cadre trop restrictif qui viendrait l'appauvrir, il convient néanmoins de porter un regard critique sur cette notion, d'en apprécier la teneur (d'aucuns diraient la saveur) afin de pouvoir mieux en préciser la pertinence.

\section{De quoi parle-t-on?}

De manière assez classique, l'apparition de nouveaux termes dans la nomenclature touristique s'accompagne d'une multiplication de définitions, lesquelles se recoupent plus ou moins, selon les disciplines qui investissent tour à tour ce champ de la réflexion. Faisons ici un rapide tour d'horizon.

De façon générale, le géotourisme renverrait aux pratiques touristiques en lien avec la découverte de la Terre. Cette entrée en matière assez large permet de regrouper dans un même ensemble des pratiques très différentes. Ces dernières peuvent aller d'un sentier de découverte des vignobles valaisans à la visite des fjords norvégiens, en passant par la descente en rappel d'un canyon dans les Alpes-Maritimes, agrémenté d'une visite d'un musée des techniques et des sciences mettant en scène une histoire minière. Cela fait-il sens de regrouper des pratiques aussi diverses dans un même ensemble "géotourisme»?

Pour certains, il semble que cela soit le cas, en atteste la définition retenue par l'American National Geographic Society : "Geotourism is defined as tourism that sustains or enhances the geographical character of a place - its environment, culture, aesthetics, heritage, and the well-being of its residents" (cité par Zorn et al., 2009:2).

Le caractère très large de cette définition limite son efficience, à tel point que l'on se demande s'il ne s'agit pas là d'un parti pris délibéré. Il semble que l'enjeu ne soit pas tant ici de proposer une définition opérationnelle que de souligner 
un changement de valeur dans les rapports homme-environnement, et ce, en lien avec le paradigme du développement durable. Le géotourisme se confond ici avec des dynamiques territoriales articulées autour du «mieux vivre ensemble». Si l'on s'en tient à cette définition englobante, presque toutes les pratiques touristiques peuvent être qualifiées de géotouristiques, dès lors qu'elles sont proposées et perçues par les acteurs comme des moyens de "valoriser la personnalité géographique d'un lieu».

À une échelle d'analyse plus fine, le développement du géotourisme s'inscrit en écho des processus de patrimonialisation des caractéristiques géologiques et géomorphologiques remarquables et de la reconnaissance des géotopes et des géosites, autrement dit de la géodiversité. De manière générale, la géodiversité regroupe l'ensemble des sous-sols, sols et paysages et renvoie tout autant aux phénomènes passés dont la Terre porte les marques qu'à des processus actuels de modification de ces mêmes paysages. L'accent est donc mis sur une double entrée géologique et géomorphologique, ainsi que sur une double échelle de temps : le temps long des héritages et le temps actuel des transformations.

Quant aux géotopes, les uns les définissent par rapport à leurs caractéristiques intrinsèques : "Les géotopes sont des formations inanimées qui illustrent l'histoire de la planète en révélant différents aspects de l'évolution de la Terre ou de la vie. Cela inclut les affleurements des roches, les sols, les minéraux et les fossiles ainsi que les formations naturelles ou les paysages» (Wuttke, 2001). D’autres préferrent une approche plus globale et définissent comme géotopes «tout objet géologique présentant une certaine valeur, qu'elle soit scientifique, historico-culturelle, esthétique ou socio-économique» (Panizza et Piacente, 2003).

Cette mise en perspective entre géotourisme et géodiversité par la notion de géotope éclaire en retour la définition de ces pratiques touristiques. Aussi, le géotourisme se définit, pour partie, comme l'ensemble des activités touristiques liées aux géotopes et aux géomorphosites, autrement dit aux formations géologiques au sens large, aux formes de reliefs et aux processus géomorphologiques remarquables.

\section{Les paysages du géotourisme}

Nombres de paysages, de types de reliefs, sont a priori des lieux où le géotourisme peut se développer. Ici, c'est une entrée par les types de paysages et de ressources qui a été retenue, laquelle conduit à une typologie de sites potentiellement géotouristiques (voir tableau 1). Pour autant, ces catégories ne sont pas exclusives les unes des autres, et de nombreux sites peuvent s'inscrire dans plusieurs d'entre elles : ainsi, le paysage culturel de Hallstatt-Dachstein/Salzkammergut, en Autriche, vaut tout autant pour le karst (en surface et sous terre) que pour le lac, pour les anciennes mines de sel et pour les glaciers du Dachstein. On remarque également que certains sites remarquables sont peu réductibles à cette typologie, comme Ayers Rock, ou Uluru, gros bloc de grès rouge isolé au centre du désert australien. On pense également aux paysages qui portent la marque de grands événements catastrophiques : éboulements rocheux, destructions liées à des éruptions volcaniques, sismicité active.

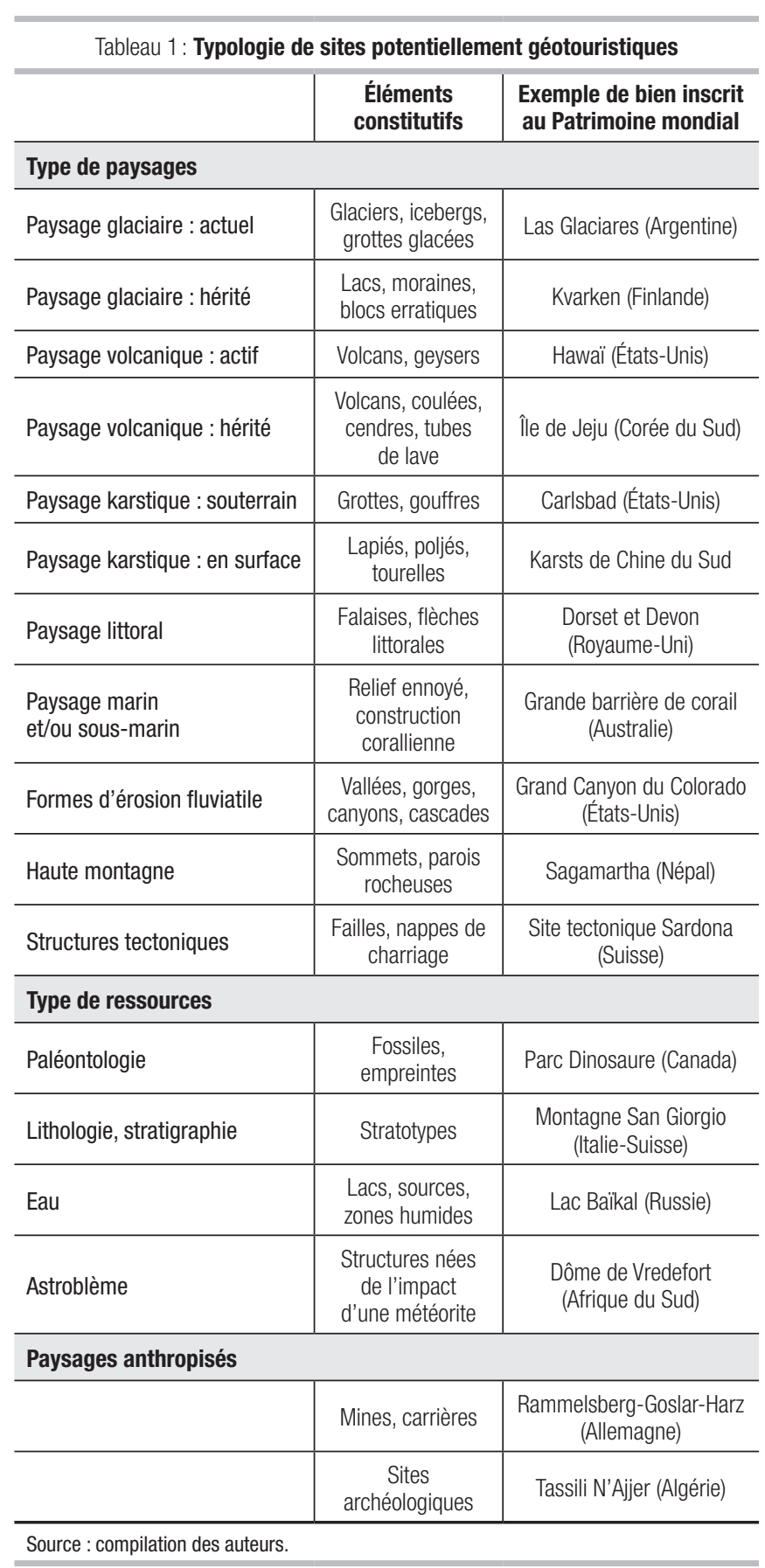

\section{La médiation scientifique comme pierre angulaire du géotourisme?}

Pour autant, cette entrée par les paysages ne semble pas suffisante pour définir le géotourisme de façon satisfaisante. Nombre de sites présentent des géopropriétés intéressantes et ne font pas l'objet de mise en tourisme (ainsi les mines encore en activité ou plus simplement des paysages de surface banalisés); ou lorsqu'il y a mise en tourisme, celle-ci ne s'organise pas nécessairement autour d'un géodiscours et la condition (sine qua 

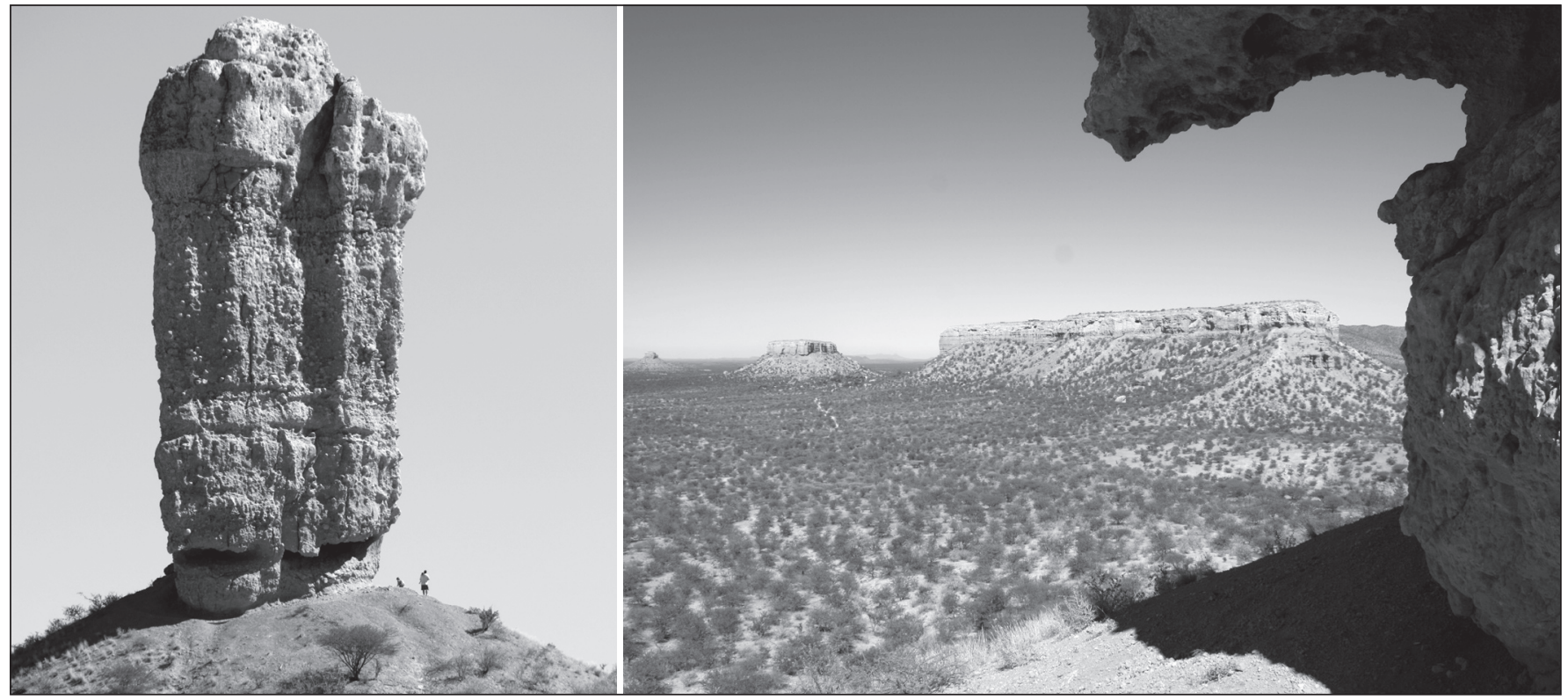

ILLUSTRATION 1 : Site monolithique de Vingerklip, Namibie (photos : Mélanie Duval).

non?) de la médiation scientifique n'est pas remplie. Nombre de sites aux caractéristiques géologiques ou géomorphologiques impressionnantes se visitent sans qu'il y ait pour autant la moindre information concernant les sciences de la Terre. Cela est vrai tant pour des géosites des pays du Nord (nombreux sites volcaniques isolés du massif central en France) que des pays du Sud. Ainsi, l'exemple du site de Vingerklip, Namibie (voir illustration 1 ) : pour un droit d'accès symbolique $(1 €)$, on est autorisé à traverser une propriété privée et à garer sa voiture au pied de ces formations surprenantes. Le seul aménagement consiste en un chemin mal entretenu qui permet d'accéder à un point de vue. Aucune forme de médiation scientifique n'est proposée. Le touriste n'a absolument aucune clef de lecture pour comprendre les paysages qu'il observe.

Hose (2005, 2006, 2008), Frey et al. (2006) et Dowling et Newsome (2006) posent clairement comme condition au géotourisme la présence d'une médiation scientifique puisqu'il s'agit pour les touristes d'acquérir des connaissances et de comprendre la géomorphologie d'un site, dépassant une première valeur esthétique : "The main tasks of geotourism are the transfert and communication of geoscientific knowledge and ideas to the general public» (Frey et al., 2006:97).

Les finalités de ce processus d'apprentissage sont doubles. S'il s'agit d'abord de garantir au touriste une expérience nouvelle, il est peut être davantage question de le sensibiliser aux particularités des géopatrimoines, d'alimenter des processus d'appropriation. Ceux-ci seront à terme les meilleurs garants d'une gestion durable du couple patrimoine-tourisme, vérifiant ainsi l'adage : À travers l'interprétation, la compréhension; à travers la compréhension, l'appréciation; à travers l'appréciation, la protection.

Comprendre plus précisément la teneur du géotourisme oblige alors à dépasser la simple question du type de lieu mis en valeur et à interroger les discours et les ressorts touristiques sur lesquels s'appuie l'expérience touristique. En vue de définir les pratiques qui relèvent du géotourisme, une des pistes de réflexion peut être de mesurer la présence ou non d'une médiation scientifique, l'importance de cette dernière ainsi que les formes qu'elle revêt. L'inscription d'une pratique touristique dans le champ du géotourisme serait ainsi directement liée (voire conditionnée) à la présence de stratégies visant à transmettre au grand public des connaissances et notions géoscientifiques.

On aurait ainsi une sorte de curseur allant de géosites touristiques dénués de géodiscours à ceux dont l'aménagement touristique s'organise exclusivement autour de la valorisation des caractéristiques géologiques et géomorphologiques.

Ainsi, l'article de Julien Meignan, dans ce numéro, démontre que pour un même objet, à savoir d'anciennes carrières exploitées en France, certaines peuvent être qualifiées comme étant des lieux du géotourisme et d'autres non, au regard des modalités de la mise en tourisme et de la place d'un géodiscours dans cette dernière. Quant au canyon des Pyrénées-Orientales étudié par André Suchet, on verra qu'il illustre le degré zéro du géodiscours!

Dans les faits, la distinction n'est pas aussi évidente et la mise en tourisme d'un géosite peut jouer sur plusieurs registres, mobilisant tour à tour des discours scientifiques, des effets de mise en scène jouant sur l'émotionnel, des éléments du folklore ou des cultures locales. Ainsi, dans la plupart des grottes touristiques en France, les visites font alterner des explications sur la genèse des cavités et des concrétionnements qu'elles présentent, le récit de légendes plus ou moins inventées qui seraient attachées au lieu, quelques plaisanteries pour scander le parcours de la visite, et pour finir, de plus en plus souvent, un spectacle son et lumière qui met en valeur un secteur particulièrement esthétique. Plusieurs discours sont ainsi tour à tour mobilisés, formant une expérience globale 

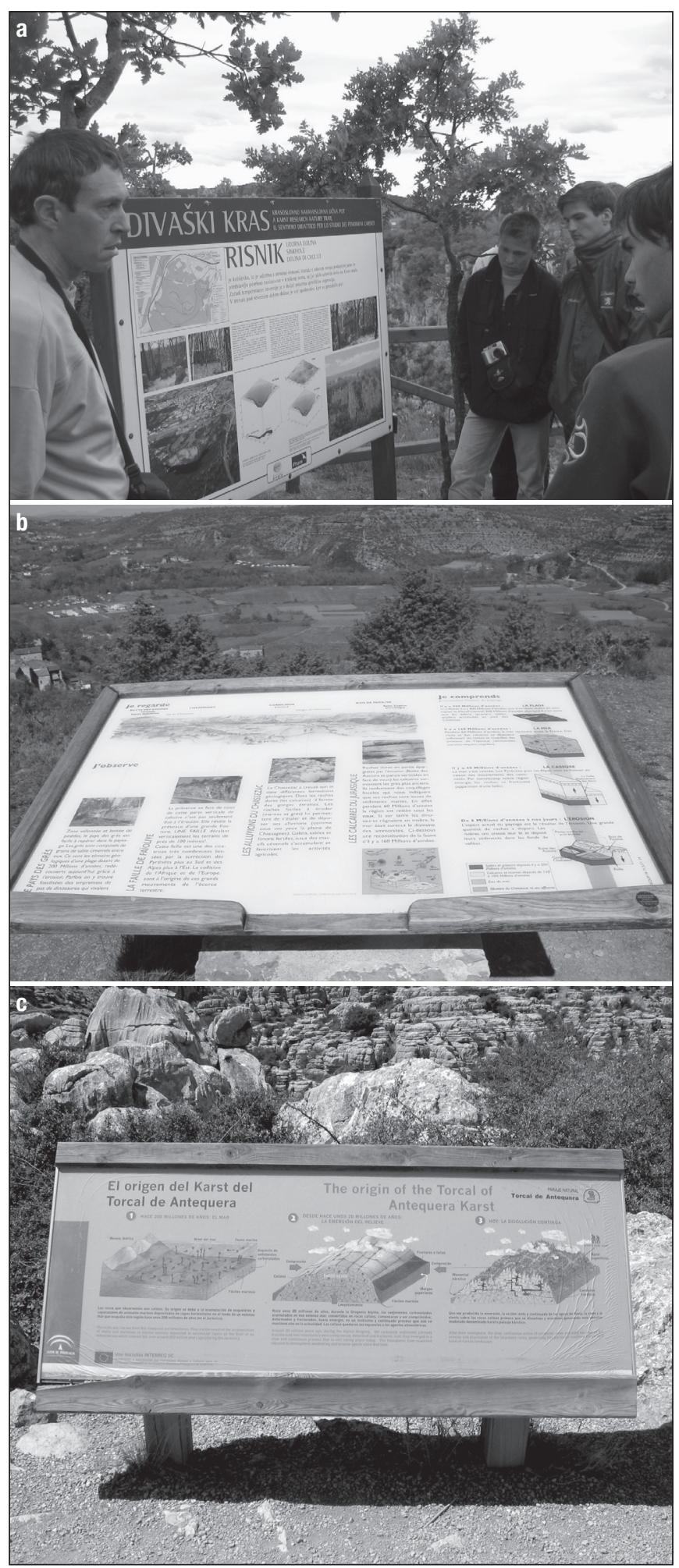

ILLUSTRATION 2 : a) Sentier géotouristique autour de la doline d'effondrement de Divača, Slovénie; b) panneau d'interprétation au niveau des gorges du Chassezac, bas Vivarais, France; c) sentier géotouristique dans le site karstique del Torcal de Antequera, Espagne (photos : Mélanie Duval). visant à satisfaire les différentes attentes du public touristique (Biot, 2006). Le géodiscours, en se combinant à d'autres types d'expériences touristiques, engendre des objets hybrides où le géotourisme est associé à d'autres dimensions.

\section{Les modalités de mise en valeur}

Les formes de mise en valeur ainsi que les supports employés en matière de médiation scientifique sont multiples. Elles peuvent être appréhendées au regard de la distance entre le lieu de l'expérience touristique et les paysages ou processus expliqués. Ainsi, deux logiques s'observent : des processus de valorisation in situ avec des aménagements au plus près du géosite, des modes de mise en valeur ex situ avec des espaces muséographiques, l'un et l'autre pouvant parfois se combiner. La valorisation in situ s'applique en particulier aux sites les plus spectaculaires (glaciers, fjords, canyon...); la dissociation spatiale s'impose au contraire pour des raisons d'accès et de protection, par exemple lorsqu'il s'agit de faunes quaternaires extraites du pergélisol sibérien. Les fossiles peuvent être montrés directement sur l'affleurement où ils ont été identifiés, mais cela nécessite des dispositifs complexes de protection; ils peuvent aussi être échantillonnés, prélevés et présentés dans un musée.

Posée ainsi, cette question du lieu amène à envisager dans un même ensemble "géotourisme» des sentiers de randonnée à la découverte des glaciers, des centres d'information dans des maisons de parcs naturels régionaux ou d'autres types d'espaces protégés contenant des géosites, ou encore des expositions sur les sciences de la Terre dans des capitales mondiales (Cité des sciences à Paris, par exemple). Pour certains auteurs, le survol en hélicoptère de site comme celui de Bungle Bungle Range (parc national de Purnululu, Australie) s'inscrit également dans le registre du géotourisme, étant donné que cette expérience touristique s'accompagne de géodiscours (Newsome et Dowling, 2006 : 8). Cela questionne, au passage, la réalité de l'adéquation entre écotourisme et géotourisme, si souvent mentionnée comme étant une évidence (Cayla, 2009). Plusieurs études de cas montrent, en effet, les risques de développer des activités touristiques dans des géosites sans que des actions de protection efficaces soient mises en place : nombre de sites paléontologiques ou fossilifères se sont ainsi vu dépouillés par les prélèvements effectués par des touristes peu conscients des conséquences de leurs actes (Hose, 2006).

S’interroger sur le géotourisme nécessite alors de prendre à la fois en considération les types de lieux dans lesquels cette activité se développe, mais également les aménagements réalisés pour satisfaire une logique de découverte, qu'il s'agisse d'infrastructures lourdes ou plus légères, nécessaires à une diffusion des connaissances géologiques et géomorphologiques à destination de différents publics touristiques : "Materials that can be used to assist in interpreting geosites include: books, displays, videos, slide shows, interactive touch panels, models, speciments, computer animations and activities" (Newsome et Dowling, 2006 : 13). Plus encore, une réflexion s'impose sur le contenu des outils de la médiation et sur la capacité qu'ont ces derniers à satisfaire les attentes des touristes, à la fois en termes d'apprentissage et de divertissement (Cayla et al., 2010). Cette réflexion, encore peu développée, a été amorcée 
par les travaux de Hose (2006) : à partir du site de Ludlow (Angleterre), il a analysé les comportements touristiques et leurs degrés de satisfaction par rapport aux supports de médiation offerts à la visite. Dans le même registre, les études de Pralong (2006) ont montré que les supports généralement utilisés dans des entreprises de médiation scientifique n'étaient pas perçus de la même manière selon les publics considérés. Dans ce numéro, Simon Martin, Géraldine Regolini-Bissig, Amandine Perret et Lenka Kozlik abordent cette question sous l'angle de l'élaboration et démontrent la nécessité qu'il y a aujourd'hui de développer des méthodologies performantes visant à évaluer les réalisations géotouristiques en vigueur et à venir (voir illustration 2).

\section{Les débuts du géotourisme}

Pour autant que ces processus de médiation renvoient a priori à des techniques contemporaines, peut-on réserver le vocable de géotourisme à des pratiques récentes?

Au fond, qu'y a-t-il de différent entre les récits de Saussure décrivant les phénomènes géologiques remarquables du massif du mont Blanc et les sentiers de découverte actuels tels que le sentier glaciologique de Champagny-en-Vanoise (voir illustration 3) ? Certes, le degré de compréhension de ces paysages n'est pas le même, les sciences de la Terre ayant fait entre temps des progrès considérables, mais la logique et les motivations qui animent le visiteur s'organisent autour de cette même volonté de comprendre et de décrypter les formes paysagères. Les différents supports de la médiation scientifique s'appuient d'ailleurs souvent sur cette continuité en faisant référence à l'histoire des sciences, à la découverte et à la compréhension du site ou de la ressource valorisée : reproduction d'anciens schémas ou d'anciennes photographies, croquis tirés des carnets de notes, fac-similé de publications pionnières...

Cependant, si le terme de géotourisme est récent, la réalité qu'il désigne n'est-elle pas aussi ancienne que le tourisme lui-même? Dans ce numéro spécial, l'article de Nathalie Cayla souligne très justement les liens qui ont existé dans le monde alpin entre les débuts du tourisme et la mise en place de formes de géotourisme. Dans un registre similaire, Hose a identifié une des origines du géotourisme dans le Peak-district au centre de l'Angleterre dès le XVIII siècle (Hose, 2008, dans Burek et Prosser, 2008 : 43-48), et Zorn et al. (2009) ont montré que l'actuelle Slovénie pouvait être tenue pour le berceau du géo-

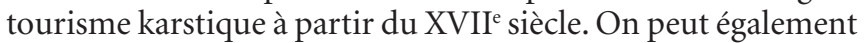
voir en Naples l'un des grands centres pionniers du géotourisme en Europe, tant les phénomènes volcaniques ont captivé les voyageurs. L'éruption du Monte Nuovo en 1538 a attiré l'attention de tous les savants de la Renaissance et, au siècle suivant, les gentilshommes qui pratiquaient le Grand Tour ne voudront pas manquer le spectacle de ces merveilles.

Lors de son premier voyage en Italie, Goethe est parvenu jusqu'à Rome. Le 24 novembre 1786, il note dans son journal :

L'éruption actuelle du Vésuve met en mouvement la plupart des étrangers ici et il faut se faire violence pour ne pas se laisser entraîner. Ce phénomène naturel a réellement quelque chose du serpent à sonnettes, il attire les hommes avec une force irrésistible. Pour l'instant il

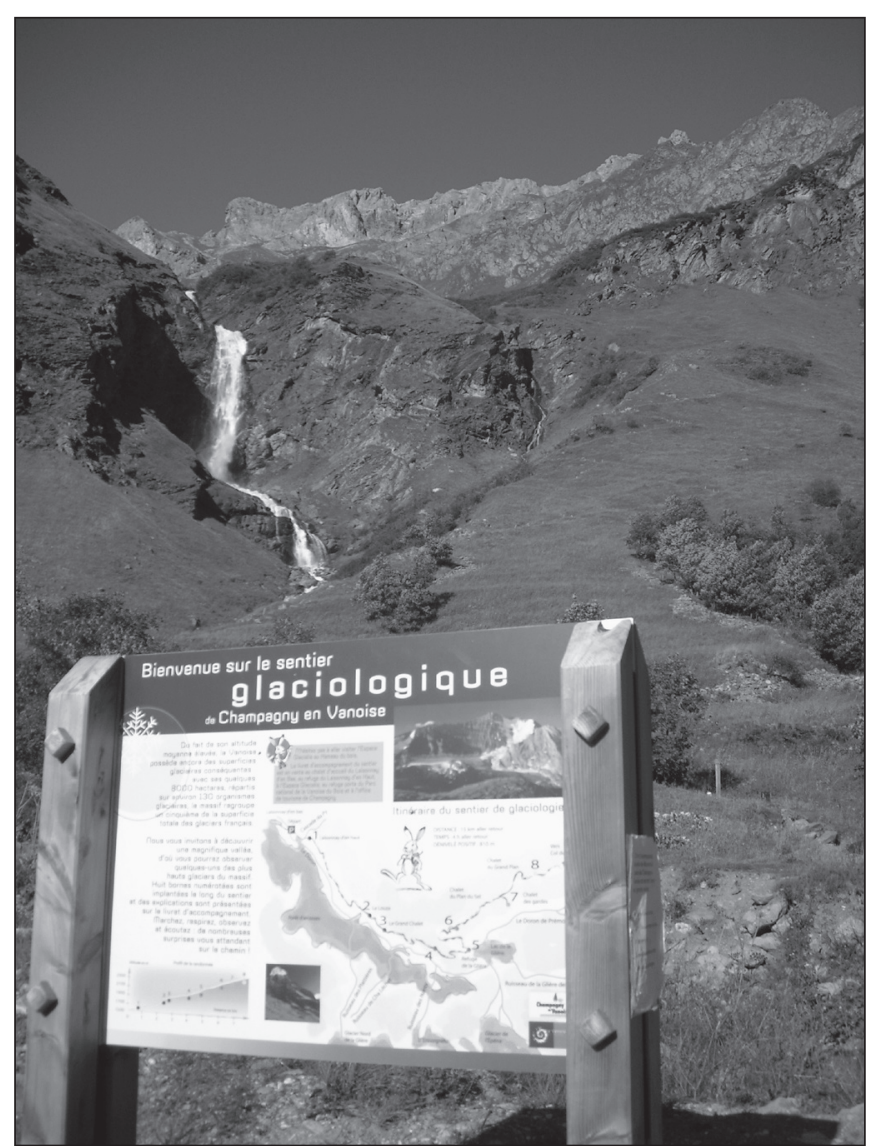

ILLUSTRATION 3 : Le sentier glaciologique de Champagny-en-Vanoise, France. Mis en place en 2007, celui-ci s'inscrit dans une volonté de développer le tourisme estival dans un espace marqué par les pratiques hivernales autour du ski alpin (photo : Mélanie Duval).

semble que tous les trésors artistiques de Rome soient réduits à rien, tous les étrangers interrompent le cours de leurs observations et se précipitent à Naples. Moi je veux persévérer ici, dans l'espoir que la montagne mettra quelque chose en réserve pour moi $(1961: 289)$.

De fait, il lui faudra patienter jusqu'à la fin de février 1787 pour se mettre en route et pour parvenir à Naples : le Vésuve l'aura attendu, et Goethe en fera l'ascension à trois reprises de façon à observer différentes phases de l'éruption au fur et à mesure que l'information lui parvient d'une nouvelle coulée de lave ou d'un autre événement remarquable. Son récit nous en dit long sur le pouvoir d'attraction géotouristique d'un phénomène exceptionnel, sur sa capacité à supplanter les ressorts classiques du tourisme d'érudition et sur les arbitrages auxquels un voyageur raisonnable doit se livrer en misant sur la poursuite de l'éruption.

Certes Goethe n'est pas un géotouriste innocent, il « voyage pour s'instruire» (1961 : 73). Pour parvenir à Rome, il a évidemment traversé les Alpes, et son récit de voyage fourmille de notations ayant trait à la géologie, aux formes du relief, aux hypothèses émises quant à sa formation... Pour lui, la géologie constitue un élément du paysage dont il convient de noter 


\begin{tabular}{|c|c|c|}
\hline \multicolumn{3}{|c|}{$\begin{array}{l}\text { Tableau 2: Biens inscrits au patrimoine mondial } \\
\text { uniquement en fonction du critère vii }\end{array}$} \\
\hline $\begin{array}{l}\text { Date d'inscription du } \\
\text { bien sur la liste Unesco } \\
\text { en raison du seul critère } \\
\text { viii. Le cas échéant, date } \\
\text { d'extension }\end{array}$ & Nom du bien & Pays \\
\hline 1987 & $\begin{array}{l}\text { Parc national des } \\
\text { volcans d'Hawaï }\end{array}$ & États-Unis d'Amérique \\
\hline 1995 & $\begin{array}{l}\text { Site fossilifère de } \\
\text { Messel }\end{array}$ & Allemagne \\
\hline 1995, 2000 & $\begin{array}{c}\text { Grottes du karst } \\
\text { d'Aggtelek et du karst } \\
\text { de Slovaquie }\end{array}$ & Hongrie, Slovaquie \\
\hline 1999 & $\begin{array}{l}\text { Parc national de } \\
\text { Miguasha }\end{array}$ & Canada \\
\hline 2000 & $\begin{array}{l}\text { Parcs naturels } \\
\text { d'Ischigualasto/ } \\
\text { Talampaya }\end{array}$ & Argentine \\
\hline 2000,2006 & $\begin{array}{l}\text { Haute Côte/Archipel } \\
\text { de Kvarken }\end{array}$ & Finlande, Suède \\
\hline 2000 & $\begin{array}{c}\text { Isole Eolie } \\
\text { (lles Eoliennes) }\end{array}$ & Italie \\
\hline 2001 & $\begin{array}{l}\text { Littoral du Dorset et de } \\
\text { l'est du Devon }\end{array}$ & $\begin{array}{c}\text { Royaume-Uni de Grande } \\
\text { Bretagne et d'Illande } \\
\text { du Nord }\end{array}$ \\
\hline 2003,2010 & Monte San Giorgio & Italie, Suisse \\
\hline 2003 & $\begin{array}{l}\text { Parc national de Phong } \\
\text { Nha-Ke Bang }\end{array}$ & Viet Nam \\
\hline 2005 & Dôme de Vredefort & Afrique du Sud \\
\hline 2005 & $\begin{array}{l}\text { Wadi Al-Hitan (la vallée } \\
\text { des Baleines) }\end{array}$ & Égypte \\
\hline 2008 & $\begin{array}{l}\text { Falaises fossilifères de } \\
\text { Joggins }\end{array}$ & Canada \\
\hline 2008 & $\begin{array}{l}\text { Haut lieu tectonique } \\
\text { suisse Sardona }\end{array}$ & Suisse \\
\hline
\end{tabular}

les changements de physionomie. Il saisit chaque occasion de montrer l'étendue de ses connaissances en la matière, à la fois par la précision de ses observations et le sérieux de ses références (il cite les sommités de la géologie de son temps : Hacquet, Ferber ou Voigt). Près du lac de Garde (12 septembre 1786), il apprécie «les plus beaux rochers calcaires propres à des études pittoresques » (Goethe, $1961: 61$ ) et à l'occasion il n'oublie pas d'échantillonner pour compléter sa collection de minéraux. Le géotourisme apparaît comme l'une des composantes qui contribuent à l'agrément et à l'intérêt du voyage, mais il en devient le but éminent lorsqu'il s'agit d'aller contempler le Vésuve en éruption!

Sans doute s'agissait-il d'une pratique très élitiste, mais pas plus que ne l'était alors le tourisme en général. Les phénomènes géologiques, les formes du relief, les particularités hydrographiques ont participé dès cette époque de ce qui constituait

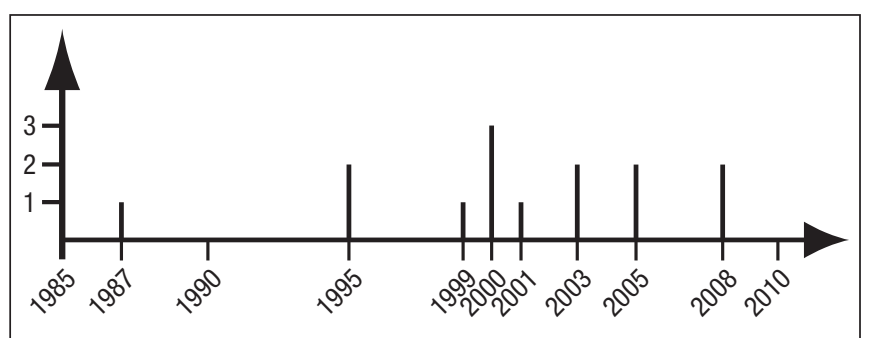

ILLUSTRATION 4 : Biens inscrits au patrimoine mondial uniquement en fonction du critère viii (source : compilation des auteurs).

la ressource touristique, aux côtés des modes de vie des populations, des monuments historiques ou des paysages végétaux.

D'un point de vue temporel, fixer une date de naissance du géotourisme n'est donc pas chose aisée. Le géotourisme renvoie-t-il réellement à une catégorie de pratiques touristiques nouvelles ou bien ne s'agit-il pas d'une requalification de pratiques anciennes? Plus simplement, le géotourisme ne signalet-il pas un changement de regard, voire de paradigme sur des espaces déjà signalés pour leur dimension pittoresque?

Sur ce point, l'évolution des outils réglementaires ainsi que l'analyse des logiques sous-jacentes aux processus de patrimonialisation d'espaces contenant des géotopes permettent de mieux comprendre et mesurer ce changement de regard.

\section{Les mesures de protection des géotopes comme indicateurs de la place occupée par la géodiversité dans les processus de patrimonialisation}

Aujourd'hui, nombres d'espaces protégés contiennent des géotopes remarquables. Pour partie, ces espaces protégés sont relativement anciens et ont constitué la première vague des sites patrimonialisés. Si on prend l'exemple français, plusieurs sites classés au titre de la loi de 1906 peuvent être considérés comme étant des géosites : ainsi, le site classé de La Pierre Bécherelle (Maine-et-Loire), classé en 1912, où sur les berges de la Loire se dresse un monolithe aux caractéristiques remarquables ou encore le site classé en 1927 des Pierres Jaûmatres (Creuse) donnant à voir un paysage de chaos granitique (Jonin et Avoine, 2010).

Pour autant, lorsqu'on reconstitue les logiques de classement, ce n'est pas tant la géodiversité qui a commandé la mise en place de telles mesures de protection qu'une volonté de préserver des caractéristiques monumentales, pittoresques, esthétiques, voire artistiques.

Graduellement, la mise en place de nouveaux espaces protégés ou encore l'inscription de nouveaux sites au patrimoine mondial de l'UNESCO vont reconnaître cette dimension «sciences de la Terre» et celle-ci sera de plus en plus invoquée.

Si l'on s'en tient à la liste du patrimoine mondial de l'UNESCO en 2010, 80 sites classés le sont en partie en raison du critère viii, c'est-à-dire "être des exemples éminemment représentatifs des grands stades de l'histoire de la [T] erre, y compris le témoignage de la vie, de processus géologiques en cours dans le développement des formes terrestres ou d'éléments géomorphiques ou physiographiques ayant une grande signification ». 
Pour la majorité d'entre eux, ce critère est associé à d'autres : que ce soit le critère vii en lien avec les caractéristiques esthétiques du site (23 sites justifient leur classement en raison uniquement de ces deux critères), le critère $i x$ renvoyant à l'importance des écosystèmes (deux sites), le critère $x$ relatif à la diversité biologique (deux sites) ou encore une combinaison entre les critères naturels (18 sites sont classés en raison des quatre critères naturels) et culturels. Si l'on observe le seul critère viii lié à la géodiversité, 14 sites sont uniquement classés en raison de ce dernier alors même que ce critère existe depuis la mise en place de la convention (UNESCO, 1977). Le premier site classé en ce sens fut le parc national des volcans d'Hawaï (1987), et, après une certaine période de latence marquée par des classements ponctuels, la tendance s'est accélérée ces dernières années (voir illustration 4 et tableau 2).

De manière assez frappante, cet état de fait souligne le décalage entre la capacité des sciences du vivant et des sciences de la Terre à valoriser leurs objets d'études : la faune et la flore ont fait l'objet de mesures visant explicitement la protection de la biodiversité depuis le milieu du $\mathrm{XX}^{\mathrm{e}}$ siècle alors que les phénomènes géologiques se sont vu accorder une valeur patrimoniale depuis peu. Moins prestigieux que les éléments du patrimoine historique, artistique ou architectural, réputés moins fragiles ou moins menacés que le patrimoine vivant, les géosites n'ont été que tardivement perçus comme patrimoniaux (Avoine et Jonin, 2010).

Ce décalage peut aussi s'expliquer par une mobilisation plus tardive des géoscientifiques et par une moindre sensibilité du public. Jusqu'à un passé récent, ni les géologues ni les géomorphologues ne se sont réellement mobilisés dans les politiques de protection de l'environnement. Ainsi, il faut attendre les années 1990 pour que la notion de géotope apparaisse alors que celle de biotope est déjà largement répandue, et ce n'est qu'à partir de ce moment-là que ce champ de réflexion et d'actions est investi par des universitaires, appuyé ensuite par le monde associatif ou par des collectivités territoriales (Cayla, 2009).

Cela explique également que, dans de nombreux parcs nationaux, les géotopes et l'entrée géodiversité restent encore secondaires par rapport aux autres caractéristiques remarquables du site. Prenant l'exemple du parc national de Yosemite, Gates (2006) rappelle que la présence d'un géodiscours est davantage perçue comme étant un bonus que comme étant la principale motivation des touristes à visiter ce genre de parc: "In general, the geological field activities are just small parts of the menu of recreational offerings of these parks, and typically they are not greatly emphasized. Clearly, the featured attraction to most of this category of parks and monuments is not the opportunity for geotourism; this is more an added bonus to help sell the park as a vacation destination» (Gates, 2006 : 162).

Toutefois on observe qu'en Europe, depuis le début des années 1990, la volonté d'inscrire des biens naturels sur la liste du patrimoine mondial se traduit souvent par le choix de géotopes : en effet, les formations végétales primaires sont rares, de même que les grandes faunes sauvages, d'où la tendance récente à privilégier les géopatrimoines.

\section{Les enjeux associés au géotourisme}

Les enjeux actuellement associés au géotourisme sont de plusieurs ordres, recoupant à la fois des logiques touristiques, patrimoniales et territoriales.

\section{Des enjeux de durabilité}

Les géopropriétés des paysages évoluent dans le temps, et le développement du géotourisme ne peut faire l'économie d'une réflexion sur la durabilité des dynamiques touristiques. En effet, s'il est une activité qui se soucie au premier plan de la qualité des paysages dans lesquels elle se développe, c'est bien le tourisme (Cazes, 1992), et surtout lorsqu'il s'agit de pratiques touristiques directement connectées à des ressorts paysagers comme c'est notamment le cas pour le géotourisme. Aussi, toute forme de développement touristique en lien avec la géodiversité appelle des mesures de protection (ou tout du moins des plans de gestion), lesquelles fonctionnent en retour comme des garde-fous et assurent pour partie les conditions de durabilité des pratiques géotouristiques.

Il est cependant des cas de figure où la mise en place de périmètres de protection et de plans de gestion ne suffissent pas à garantir la durabilité de telles pratiques touristiques : c'est notamment le cas pour des paysages particulièrement sensibles et réactifs aux changements climatiques, tels des paysages maritimes avec l'exemple des barrières de corail (James et al., 2006; Prosser et al., 2010) ou encore les paysages glaciaires. Dans le cas où ces paysages sont supports d'activités géotouristiques, ces évolutions pourraient se révéler être, à terme, autant de facteurs de vulnérabilité, remettant en question la durabilité du système touristique, voire même de systèmes territoriaux selon l'importance du secteur touristique. Tel est le cas des milieux polaires, illustré ici par l'article de Michael Hall et Jarkko Saarinen ou, à une autre échelle, par des grottes glacées des Alpes autrichiennes.

Ces contraintes extérieures poussent les systèmes touristiques à penser de nouveaux modes de mise en valeur ou, à défaut, de nouveaux registres de médiation. Des sites vont même jusqu'à construire leur médiation scientifique autour de ces processus paysagers évolutifs : ainsi, le sentier du glacier de Morteratsch (Suisse) met en évidence les effets du réchauffement climatique actuel sur le glacier (voir l'article de Nathalie Cayla, dans ce numéro).

Cette réflexion en matière de durabilité se double, implicitement, d'un questionnement sur la visibilité : en ce qui concerne les paysages englacés, pour encore combien de temps sera-t-il possible de les observer? Certains opérateurs touristiques en font d'ailleurs leurs choux gras et n'hésitent pas à jouer la carte de l'urgence à aller voir ces paysages avant qu'ils ne disparaissent, alimentant en cela des boucles de rétroactions négatives avec des impacts croissants sur les milieux polaires.

\section{Des enjeux de diffusion de la connaissance scientifique et d'appropriation}

De manière plus générale, cette question de la visibilité est valable pour l'ensemble des géotopes : dans un paysage à la géologie et à la géomorphologie complexes, que voit-on? Et surtout, dans une perspective de développement d'une 
activité géotouristique, quels sont les éléments identifiables, appréhendables et compréhensibles pour un touriste, qu'il soit connaisseur ou néophyte?

Sur ce point, des décalages s'observent entre des géotopes patrimonialisés et la possibilité pour les touristes de s'approprier les formes protégées : c'est, par exemple, le cas du chevauchement de Glaris, classé au patrimoine mondial de l'UNESCO en 2008 sous l'appellation du «Haut lieu tectonique suisse Sardona ». La justification du classement repose sur le seul critère de la géodiversité :

Histoire de la Terre, éléments et processus géologiques et géomorphiques : Le Haut lieu tectonique suisse Sardona présente une exposition exceptionnelle de tectorogenèse et est reconnu comme un site capital pour la géologie depuis le $18^{\text {ème }}$ siècle. L'exposition claire du chevauchement de Glaris est une caractéristique clé, sans être la seule caractéristique importante. Les expositions de roches, au-dessus et au-dessous de cette caractéristique, sont visibles en trois dimensions et, ensemble, ont apporté des contributions importantes à la connaissance de la tectorogenèse. Ses caractéristiques géologiques peuvent être facilement appréciées par tous les visiteurs. Le bien peut être distingué d'autres sites semblables par l'association de l'exposition claire du phénomène dans un cadre montagneux, l'histoire de son étude et sa contribution permanente à la géologie (UNESCO, 2008).

Néanmoins, pour autant que ce site ait été et continue à être capital pour la compréhension de la formation des Alpes, dans quelle mesure le développement d'une activité géotouristique permet-elle aux touristes de s'approprier ces géoréalités? Sur le terrain, il n'est pas toujours aussi facile de voir le chevauchement que sur les nombreuses photos aériennes qui documentent le site.

De manière forte, un des enjeux du géotourisme réside alors dans les modalités de mise en valeur et dans la qualité de la médiation scientifique: «Because rocks and landscapes are not alive in the sense that plants and animals are, geotourism is particularly reliant on explanation of origin and meaning. With appropriate interpretation, any landscape, rock outcrop or landfrom can be made as exciting as spectacular displays of wildflowers or concentrations of wild animals " (Newsome et Dowling, 2006 : 14). Ces enjeux de médiation et d'appropriation posent également la question de la formation des guides, personnes-ressources dans les processus d'apprentissage associés au géotourisme : dès lors que des notions géotouristiques sont intégrées à la visite, les guides doivent recevoir une formation spécifique. Cet aspect reste aujourd'hui relativement peu étudié et pourtant, en termes de développement du géotourisme, il est central : comme pour d'autres formes de tourisme, le guide conditionne, pour partie, la réussite de l'expérience touristique (Randall et Rollins, 2009).

Ce travail de formation et de médiation scientifique est d'autant plus nécessaire qu'il conditionne en grande partie des processus d'appropriation/satisfaction des publics touristiques, permettant en cela de générer des retombées économiques.

\section{Des enjeux de diversification touristique/ allongement de la saison touristique}

À l'instar de toute "nouvelle» forme de tourisme (ou tout du moins, présentée comme telle), le géotourisme apparaît pour les acteurs comme un moyen de diversifier les ressources touristiques d'un espace donné. Plusieurs stratégies peuvent s'observer : à l'échelle du lieu, certains acteurs investiront de nouveaux lieux en vue de créer un produit géotouristique (création de sentiers d'interprétation), d'autres chercheront à faire évoluer des produits touristiques pré-existants en intégrant des éléments de géodiscours, le géotourisme participant en cela au renouvellement du produit touristique selon les cycles de vie proposés par Butler (1980). En lien avec la montée en puissance du développement durable, nombre de sites initialement aménagés dans une logique ludorécréative incorporent peu à peu les éléments d'un géodiscours. C'est par exemple le cas de la grotte de Postojna (Slovénie) : ouverte au tourisme dès le début du XIX ${ }^{\mathrm{e}}$ siècle, le contenu des visites était essentiellement axé sur le caractère fantastique, voire fantasmagorique des formations karstiques. Peu à peu, un géodiscours a été intégré, en lien direct avec l'avancée des connaissances scientifiques et la structuration de la science karstologique (Duval, 2007; Kranjc, 2004). Les lieux touristiques peuvent également présenter un certain nombre d'avantages pratiques qui permettent d'en faire aussi des lieux de recherche scientifique, les résultats de ces recherches venant à leur tour alimenter le géodiscours qui sera proposé aux touristes. Les grottes touristiques en offrent de nombreux exemples (Jaillet et Perrette, 2006). Enfin, des éléments de géodiscours apparaissent progressivement dans la visite de monuments historiques et de villes, dès lors que l'on peut éclairer la provenance des matériaux de construction par la géologie régionale : la collection «Balades géologiques en ville», éditée par la Société géologique de France, comporte déjà quatre titres (Bordeaux, Etampes, Milly-la-Forêt et la Rochelle).

À l'échelle non plus du lieu touristique mais d'une région plus vaste, les acteurs peuvent également se saisir du registre du géotourisme pour diversifier les activités touristiques présentes en vue d'attirer de nouvelles clientèles, mais également d'allonger la saison touristique : "Geotourism can extend the tourist season, as it is not entirely weather-dependent, and can bring real economic benefits to a tourist destination area» (Keever et al., 2006).

L'enjeu est à la fois de sensibiliser les visiteurs à la géodiversité, mais également, dans une logique touristique, d'augmenter le temps passé sur le site et donc, de manière implicite, les dépenses effectuées (Frey et al., 2006).

Les enjeux économiques associés au géotourisme peuvent, en effet, être majeurs à l'échelle locale. Pour s'en donner une idée, on peut se référer à des études conduites sur les sentiers à thème, et notamment celle menée par $\mathrm{M}$. Rohn-Brossard (2006). Basée sur l'analyse d'une dizaine de sentiers à thème répartis sur l'ensemble du territoire suisse (les sentiers retenus ne relèvent pas nécessairement du géotourisme), cette recherche a montré que les retombées économiques pour le territoire peuvent osciller entre des extrêmes, allant de retombées économiques quasiment nulles à des retombées importantes. Ainsi, pour 1 CHF investi, ces retombées directes peuvent 
être de l'ordre de 0,3 CHF, comme c'est le cas pour un sentier d'interprétation en lien avec l'activité agricole à Zollbrück (canton de Berne), mais elles peuvent également être 50 fois supérieures au montant de l'investissement comme c'est le cas pour le sentier de Muggestutz, à Halisberg, canton de Berne. Ces différences s'expliquent à la fois en raison des montants initialement investis, des taux de fréquentation touristique, mais également des plans de développement touristique, des stratégies de communication, etc. (Cayla, 2009 : 110).

Les retombées économiques associées au géotourisme, qu'elles soient réelles, supposées ou fantasmées, expliquent la mobilisation actuelle des acteurs territoriaux autour de cette entrée géotouristique.

\section{Des enjeux de territoire}

De manière de plus en plus nette, les dynamiques géotouristiques se doublent d'enjeux territoriaux : autour de paysages aux caractéristiques géologiques et géomorphologiques scientifiquement et socialement reconnues comme étant exceptionnelles, des projets de territoire apparaissent. Cela est particulièrement visible à travers l'exemple des géoparcs. Ces derniers se sont progressivement mis en place à partir des années 1990, d'abord à une échelle européenne avec le Réseau European Geoparks créé en 2000 (Cayla, 2009 : 38), puis mondiale avec le Réseau mondial des géoparcs soutenu par l'UNESCO, établi en 2004 (Cayla, 2009 : 33). Pour faire partie de ces réseaux, un espace candidat doit démontrer qu'il est : un territoire aux limites bien définies qui a une superficie assez étendue pour contribuer au développement économique local. Il comprend un certain nombre d'héritages géologiques (à différentes échelles) ou une mosaïque d'entités géologiques d'importance scientifique particulière, pour leur rareté ou leur beauté représentative d'un lieu et de son histoire géologique. Il ne doit pas seulement avoir une signification géologique mais aussi écologique, archéologique, historique ou culturelle (Eder et Patzak, 2004, cité dans Cayla, 2009 : 34).

Le géoparc témoigne ainsi du passage d'une logique de collection de sites à une logique de cohérence territoriale autour du géopatrimoine.

Si l'on essaie de faire une synthèse de la littérature existant à ce sujet (Zouros, 2005; Frey et al., 2006; Cayla, 2009; Martini, 2010), les objectifs des géoparcs sont essentiellement de cinq ordres :

1- la préservation des géopatrimoines;

2- le transfert des connaissances auprès d'un large public par une offre touristique appropriée;

3- la mise en placed'une économie durable autour de cette double entrée préservation/valorisation des géopatrimoines;

4- l'identification de la société locale à la ressource valorisée;

5- la mise en réseau des différents acteurs.

Ainsi, du point de vue des acteurs du territoire et de l'ancien directeur de la réserve naturelle géologique de HauteProvence, "avec les Géoparcs, nous sommes passés d'une problématique d'inventaire du patrimoine géologique, d'un regard porté sur des sites ponctuels et isolés à la définition de territoires cohérents à dominante géologique et de par cette spécificité nous avons développé des politiques économiques basées sur le développement durable» (Martini, 2010 : 36).

Dans les milieux ruraux, les géoparcs sont dès lors perçus par les acteurs comme un moyen d'insuffler de nouvelles dynamiques de développement socio-économiques, que ce soit dans le contexte des pays en voie de développement où les campagnes se situent généralement en marge des circuits économiques (Reimold et al., 2006) ou dans celui des pays occidentalisés où les espaces ruraux font figure de territoires vieillissants.

Cela est particulièrement le cas dans des territoires marqués par une rurbanisation éclatée comme dans les communes du Chablais : entre des stations de ski et la métropole lémanique, les communes rurales peinent à trouver leur place. Dans le contexte actuel de mise en concurrence des territoires, la démarche géoparc est alors envisagée par les acteurs comme un moyen de se fédérer, de créer une lisibilité territoriale autour des entrées géopatrimoniale et géotouristique, et implicitement d'attirer de nouveaux types de financement nécessaires à la revitalisation de leurs territoires. Ces perspectives aboutissent aujourd'hui à une multiplication des candidatures, au risque d'une banalisation du label.

Par un effet rétroactif, de telles démarches viennent alimenter la recherche scientifique : créer une identité territoriale autour d'une entrée "géodiversité» suppose des démarches d'inventaire systématique des géoressources présentes sur le territoire considéré. Ces démarches permettent d'approfondir la connaissance d'un territoire et soulignent les partenariats vertueux qui peuvent s'établir entre la communauté scientifique et les édiles locaux. De telles dynamiques s'observent dans de nombreux cas de figure, que ce soit dans le cadre de la réserve naturelle géologique de Haute-Provence (Guiomar et al., 2010) ou encore dans des projets de géoparc en Malaisie, dans l'état de Sarawak (Tongkul, 2006).

Les dynamiques territoriales autour d'une entrée géotouristique ne sont pas l'exclusivité des réseaux géoparcs et se retrouvent dans d'autres types de démarches, tels que des projets de classement UNESCO ou encore la mise en place de parcs naturels régionaux. Ainsi la question se pose sur d'anciens sites miniers confrontés à une reconversion, lorsqu'il s'agit de donner du sens à une activité minière recouvrant de forts enjeux identitaires tout en permettant de trouver de nouvelles voies de développement économique. Par une mise en scène du patrimoine local, l'entrée géotouristique, pensée dans un cadre territorial, permet d'assurer la transmission de la mémoire de la mine et de dépasser le traumatisme de territoires qui, du jour au lendemain, ont vu l'arrêt de leur principale activité économique. À ce titre, les mines d'Idrija, en Slovénie, se sont engagées dans un processus de valorisation géotouristique de leur patrimoine minier lié à l'extraction du mercure depuis le XVI ${ }^{\mathrm{e}}$ siècle. Pilier central dans l'économie de la région pendant plusieurs siècles, cette activité s'est progressivement réduite depuis les années 1980, en lien avec l'évolution des normes environnementales. La décision européenne de stopper toute extraction du mercure en 2001 a entraîné la fermeture définitive des mines en 2003. Ainsi, les mines d'Idrija se sont retrouvées confrontées à des enjeux de reconversion importants : que faire de ce patrimoine millénaire? Comment 


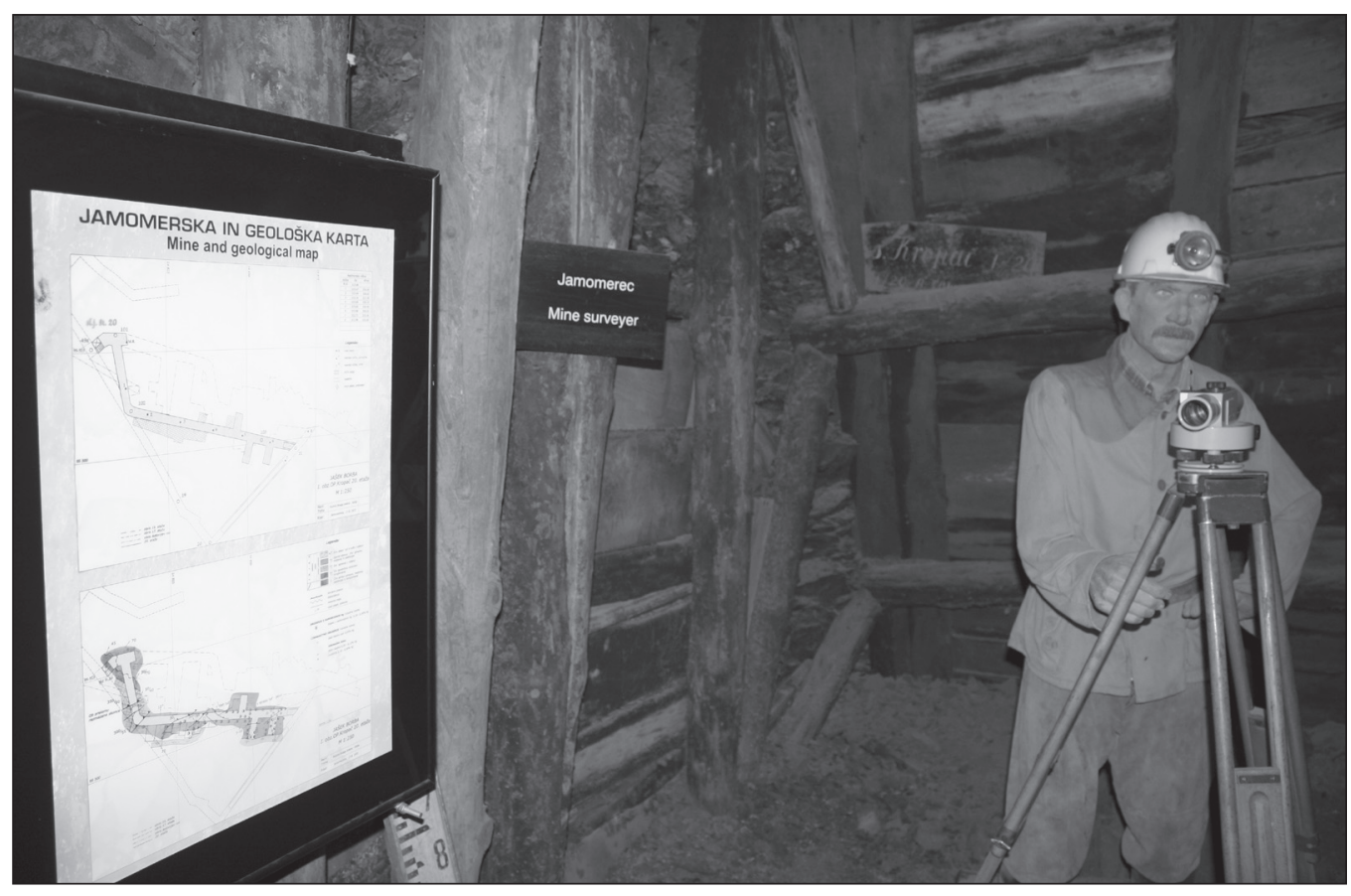

\section{ILLUSTRATION 5 :}

Les mines d'Idrija, Slovénie (photo : Mélanie Duval). gérer et donner à voir l'histoire singulière de cette région? Comment générer de nouvelles retombées économiques pour ce territoire?

Ouvert en 1995, la partie touristique des mines d'Idrija permet de visiter une partie des galeries où s'effectuait l'extraction du minerai du mercure. Les registres de la visite alternent entre la présentation de l'histoire des sciences, des caractéristiques géologiques, des techniques d'extraction et des conditions de travail dans la mine (voir illustration 5). Autour de cette visite touristique et d'un musée des sciences et des techniques, des stratégies territoriales se sont mises en place avec notamment une demande de classement au patrimoine mondial de l'UNESCO. Le projet consiste à inscrire un bien sériel dénommé «Mercury Route of the Intercontinental Camino Real», en lien avec les mines de mercure d'Almadèn en Espagne et les mines d'argent de San Luis Potosí (Mexique). Ces trois sites sont présentés comme des sites majeurs pour comprendre l'évolution des techniques et la mise en place d'un système économique mondial, le mercure extrait des mines européennes étant alors acheminé au Mexique pour servir à l'amalgamation de l'argent.

À l'heure actuelle, il semble que ce projet d'inscription transatlantique ait été ajourné, le Mexique ayant vraisemblablement préféré faire cavalier seul pour l'inscription UNESCO. Récemment obtenue en juin 2010, le bien «Camino Real de Tierra Adentro" reste un bien sériel englobant plusieurs sites majeurs pour l'extraction et la transformation de l'argent, mais il se situe uniquement sur le territoire mexicain. De plus, l'accent porte désormais sur des aspects historiques et architecturaux, la valorisation de ce bien sortant par-là même du registre géotouristique.

À l'échelle du site d'Idrija, ce retrait (temporaire?) de la démarche UNESCO ne change pas pour autant la donne, le site continuant de fonctionner autour du musée et de la visite de la mine. Le principal apport de cette démarche est davantage à comprendre en termes de gouvernance. Ainsi, aux dires des acteurs d'Idrija, cette démarche leur a permis de travailler ensemble et de mettre en place des bases solides pour les collaborations à venir, lesquelles pourraient d'ailleurs prendre la forme... d'un géoparc!

Du point de vue des acteurs, le géotourisme se situe à la croisée de différents registres et domaines de compétences : les services de la protection de la nature tout comme les acteurs culturels peuvent être impliqués dans des dynamiques géotouristiques. À cela s'ajoutent les professionnels du tourisme, les élus locaux, les populations locales, etc. Au final, une kyrielle d'acteurs, aux attentes et projections parfois différentes, s'observe autour de projets géotouristiques. Un des enjeux en matière de géotourisme réside très précisément dans la capacité que ces derniers auront à travailler ensemble : " $[T]$ o be successful, conservation of geological heritage requires the concerted involvement of all stakeholders, both geologists and non-geologists (e.g. politicians, planners, landowners, developers and the public/local communities)»(Tongkul, 2006:40).

On comprend que le géotourisme mobilise de multiples notions culturelles, au-delà des seules connaissances géologiques. Ce sont les représentations de la nature qui sont en jeu, telles qu'elles ont été transmises et telles qu'elles se transmettent encore à travers les pratiques touristiques : l'article de Benjamin Taunay est à cet égard très instructif pour les comparaisons qu'il opère entre la vision des karsts de Chine du Sud par les touristes chinois et par les touristes étrangers. La recherche à venir sur le géotourisme (et sur la gestion de la géodiversité de manière générale) gagnera certainement à mieux prendre en compte cette dimension culturelle au sens large (Hobléa, 2009). Rappelons qu'Uluru-Ayers Rock avait 
été inscrit sur la liste du patrimoine mondial en 1986 au titre des biens mixtes, juxtaposant une dimension naturelle et une dimension culturelle; en 1993, il a été requalifié en paysage culturel, nouvelle catégorie apparue alors pour mieux mettre en lumière les interactions profondes entre le site dans sa dimension géologique et paysagère, et la cosmogonie des aborigènes (Audet, $1997: 70-71$ ).

La durabilité des dynamiques géotouristiques passe, dès lors, par un travail de mise en réseau et de collaboration entre ces différents types d'acteurs. Cette question est abordée dans ce numéro par l'article de Christel Venzal, laquelle s'interroge plus particulièrement sur les conditions de durabilité des partenariats. Pour cela, un travail de clarification doit être effectué en amont, afin de s'assurer que l'ensemble des acteurs en présence partagent un même projet de territoire : «Due to communication problems, the interaction between academics and politics usually results in direct conflict for the local actors in the geotourism sector. In spite of using the same terms and motives primarily, this is due either to a lack of objectives or to having disparate objectives» (Frey et al., $2006: 98$ ).

De manière très claire, les acteurs d'un même territoire n'envisagent pas tous la valorisation des géotopes sous le même angle : alors que pour certains l'attention se porte sur leur protection et la mise en place d'une gestion durable et raisonnée, autrement dit patrimoniale, d'autres perçoivent les géotopes avant tout comme des ressources premières qu'il convient d'exploiter de la manière la plus efficace (et surtout la plus rentable!) possible. Ainsi, si l'on reprend l'exemple du projet de géoparc du Chablais, les jeux d'acteurs et les logiques d'arbitrage soulignent clairement les distorsions qu'il peut $y$ avoir entre les acteurs : dans la phase de démarrage du projet, le maire d'Excenevex (Haute-Savoie) a dans un premier temps soutenu le projet, car il y voyait le moyen d'allonger la saison touristique, de sortir d'une monoactivité et d'attirer de nouveaux types de clientèle. Sa commune, située sur les bords du Léman, possède, en effet, une plage qui a la double particularité d'accueillir plusieurs milliers de touristes chaque année et de présenter des géoprocessus remarquables avec la formation de dunes. Mettre en valeur les processus dunaires avec un fort affichage géopatrimonial aurait permis de diversifier les activités touristiques sur la commune. Par contre, cela aurait également signifié une perte de mètres carrés utiles sur la plage. Pour conclure, à la valorisation de ce géopatrimoine, le maire a clairement préféré maintenir une forte densité de serviettes de plages au mètre carré pendant la saison estivale et s'est retiré du projet de géoparc en 2009. Cet exemple souligne au passage l'importance du registre des représentations associées au géotourisme. Dépassant le seul registre économique, la position d'un acteur est également liée à l'image qu'il associe au géotourisme, aux vertus territoriales positives qu'il prête (ou non) au secteur géotouristique. En termes de jeux d'acteurs, de mise en réseaux, de collaboration et de partenariats, la question des représentations et des projections des uns et des autres est essentielle et ne peut être oubliée.

Au final, le géotourisme s'inscrit au cœur de logiques multiples, croisant des enjeux de préservation, de développement touristique, d'éducation à l'environnement, de création de lien social et identitaire, autant de facettes d'une volonté de «mieux vivre ensemble». La définition la plus appropriée du géotourisme ne devrait-elle pas alors combiner la dimension culturelle, esthétique et sociale énoncée dans la définition de l'American National Geographic Society et l'exigence d'un véritable contenu géoscientifique transmis au public? C'est là sans doute la condition nécessaire pour faire du géotourisme une composante à part entière du tourisme culturel et patrimonial. Les articles retenus pour ce numéro spécial (annoncés dans cette introduction) viendront tour à tour éclairer les différentes facettes du géotourisme et essaieront de circonscrire peu à peu les significations et les portées de ce vocable.

\section{Références}

AUDET, C. (1997) «Le classement des paysages culturels en patrimoine mondial», DANS Sciences sociales et espaces protégés, J.-L. LAURENT (dir), séminaire d'Aussois, 154 p.

AVOINE, Jacques et Max JONIN (2010) «Réserves naturelles et patrimoine géologique», Géologie de la France, nº 1, p. 11-17.

BIOT, Vincent (2006) «Le tourisme souterrain en France», Karstologia Mémoires, $\mathrm{n}^{\circ} 15$, éd. à compte d'auteur, 236 p.

BUREK, Cynthia V. et Colin D. PROSSER (éd.) (2008) The History of Geoconservation, Londres : Geological Society. 312 p.

BUTLER, Ron W. (1980) «The concept of a tourist area cycle of evolution: implications for management of resources ", The Canadian Geographer, XXIV-1, p. 5-12.

CAYLA, Nathalie (2009) «Le patrimoine géologique de l'arc alpin. De la médiation scientifique à la valorisation géotouristique». Thèse de doctorat des sciences de la Terre, de l'univers et de l'environnement, Savoie : Université de Savoie, École Doctorale SISEO. 307 p.

CAYLA, Nathalie; Fabien HOBLÉA, et Dominique GASQUET (2010) «Guide des bonnes pratiques de médiation des géosciences sur le terrain », Géologie de la France, n 1, p. 47-55.

CAZES, Georges (1992) Fondements pour une géographie du tourisme et des loisirs, Paris : Bréal. 199 p.

DOWLING, Ross et David NEWSOME (2006) «Geotourism's issues and challenges ", DANS Geotourism, Ross K. DOWLING et David NEWSOME (éd.), p. 242-254. Londres : Elsevier Butterworth Heinemann.

DUVAL, Mélanie (2007) «Dynamiques spatiales et enjeux territoriaux des processus de patrimonialisation et de développement touristique. Étude comparée des gorges de l'Ardèche et du Karst slovène». Thèse de doctorat de géographie, Savoie : Université de Savoie. 514 p.

EDER, Wolfgang et Margarete PATZAK (2004) «Geoparks — geological attractions: a tool for public education, recreation and sustainable economic development », Episodes, 27/3, p. 162-164.

FERNANDA de LIMA, Flava; José B. BRILHA. et Eduardo SALAMUNI (2010) «Inventorying Geological Heritage in Large Territories: A Methodological Proposal Applied to Brazil», Geoheritage, <http://www. springerlink.com/content/g8437115585803n2/>.

FREY, Marie-Luise; Klaus SCHÄFER; Georg BÜCHEL et Margarete PATZAK (2006) "Geoparks - a regional, European and global policy», DANS Geotourism, Ross K. DOWLING et David NEWSOME (éd.), p. 95-117. Londres : Elsevier Butterworth Heinemann.

GATES, Alexander E. (2006) «Geotourism : a perspective from the USA», DANS Geotourism, Ross K. DOWLING et David NEWSOME (éd.), p. 157-179. Londres : Elsevier Butterworth Heinemann.

GOETHE, Johann Wolfgang (1961) Voyage en Italie (éd. bilingue), 2 vol., Paris : Aubier. 792 p. 
GUIOMAR, Myette; Marc FLOQUET et Bernard BARTOLINI (2010) «De l'inventaire à la valorisation des sites paléontologiques : l'exemple du gisement à ichthyosaure de la Mélaie à Prads Haute-Bléone (Réserve naturelle géologique de Haute-Provence) ", Géologie de la France, n 1, p. 101-111.

HOBLÉA, Fabien (2009) «Karstic geomorphosites: managing subterranean natural-cultural heritage sites», DANS Geomorphosites, Emmanuel REYNARD, Paola CORATZA et Géraldine REGOLINI-BISSIG (éd.), p. 189-200. München : Verlag Dr. Friedrich Pfeil.

HOSE, Thomas A. (2005) «Geotourism : appreciated the deep time of landscapes», Niche tourism : contemporary issues, trends and cases, Londres : Butterworth-Heinemann, p. 27-28.

HOSE, Thomas A. (2006) «Geotourism and interpretation», DANS Geotourism, Ross K. DOWLING et David NEWSOME (éd.), p. 221-241. Londres : Elsevier Butterworth Heinemann.

HOSE, Thomas A. (2008) «Towards a history of geotourism: definitions, antecedents and the future», DANS The history of geoconservation, Cynthia V. BUREK et Colin D. PROSSER (éd.), p. 37-60.

JAILLET, Stéphane et Yves PERRETTE (2006) «La grotte touristique, objet de recherche et lieu de médiation", dossier «Grottes touristiques», Espaces, tourisme \& loisirs, $\mathrm{n}^{\circ} 236$, p. 33-35.

JAMES, Jane; Ian CLARK et Patrick JAMES (2006) «Geotourism in Australia», DANS Geotourism, Ross K. DOWLING et David NEWSOME (éd.), p. 63-77. Londres : Elsevier Butterworth Heinemann.

JONIN, Max et Jacques AVOINE (2010) «Sites classes et patrimoine géologique», Géologie de la France, n 1, p. 19-23.

KEEVER, Patrick; Jonathan LARWOOD et Alan McKIRDY (2006) "Geotourism in Ireland and Britain», DANS Geotourism, Ross K. DOWLING et David NEWSOME (éd.), p. 180-197. Londres : Elsevier Butterworth Heinemann.

KRANJC, Andrej (2004) Short history of cave tourism in Slovenia: 4th International ISCA Congress, Use of modern technologies in the development of caves for tourism (Postojna : 21-27 octobre 2002), p. 55-60.

LOVELOCK, James (1986) La terre est un être vivant: l'hypothèse Gaïa, Monaco : Le Rocher. 183 p.

MARTINI, Guy (2010) «Les Géoparcs pour une évolution du concept de territoire», Géologie de la France, n 1, p. 35-40.

NEWSOME, David et Ross K. DOWLING (2006) «The scope and nature of géotourisme», DANS Geotourism, Ross K. DOWLING et David NEWSOME (éd.), p. 3-25. Londres : Elsevier Butterworth Heinemann.
PANIZZA, Mario et Sandra PIACENTE (2003) Geomorfologia culturale, Bologne : Pitagora Editrice. 350 p.

PRALONG, Jean-Pierre (2006) «Géotourisme et utilisation de sites naturels d'intérêt pour les sciences de la Terre : les régions de Crans-MontanaSierre (Valais, Alpes suisses) et Chamonix-Mont-Blanc (Haute-Savoie, Alpes françaises)». Thèse de doctorat présentée à la faculté des géosciences et de l'environnement, Lausanne : Université de Lausanne. 224 p.

PROSSER, C. D.; C. V.BUREK; D. H. EVANS, ; J. E. GORDON; V. B. KIRKBRIDE; A. F. RENNIE et C. A. WALMSLEY (2010) «Conserving Geodiversity Sites in a Changing Climate: Management Challenges and Responses", Geoheritage, <http://www.springerlink.com/content/ un19014604352068/>.

RANDALL, Carleigh et Rick B. ROLLINS (2009) «Visitor perceptions of the role of tour guides in natural areas», Journal of Sustainable Tourism, 17:3, p. 357-374.

REIMOLD, Wolf U.; Gavin WHITFIELD et Thomas WALLMACH (2006) "Geotourism potential of southern Africa», DANS Geotourism, Ross K. DOWLING et David NEWSOME (éd.), p. 42-62. Londres : Elsevier Butterworth Heinemann.

ROHN-BROSSARD, Martine (2006) «Parc naturel, chemin à thème et impact économique, travail de diplôme de formation continue en écologie et en sciences de l'environnement». Mémoire de maîtrise, Neuchâtel : Université de Neuchâtel. 91 p.

TONGKUL, Félix (2006) «Geotourism in Malaysian Borneo », DANS Geotourism, Ross K. DOWLING et David NEWSOME (éd.), p. 26-41. Londres : Elsevier Butterworth Heinemann.

UNESCO (1977) Operational guidelines for the implementation of the world heritage convention, $11 \mathrm{p}$.

UNESCO (2008) Fiche " Haut lieu tectonique suisse Sardona», <http://whc. unesco.org/fr/list/1179>, consulté le 18 novembre 2010.

WUTTKE, M. (2001) «Geotope protection in the Federal Republic of Germany", DANS A future for fossils, H. G. BASSET et al. (éd.), p. 39-41. Cardiff : National Museums \& Galleries of Wales.

ZORN, Matija; Bojan ERHARTIČ et Blaz KOMAC (2009) «La Slovénie, berceau du géotourisme karstique», Karstologia, n 54, p. 1-10.

ZOUROS, Nikolaus (2005) «Assessment, protection, and promotion of geomorphological and geological sites in the Aegean area », Grèce, Géomorphologie, nº 3, p. 227-234.

\section{MERCI À UN AMI DE TÉOROS, MATHIEU DORMAELS}

La direction de la revue Téoros souhaite remercier chaleureusement Mathieu Dormaels qui a agi, jusqu'à tout récemment, comme adjoint à la rédaction, publicité et promotion de Téoros. Le doctorant s'est joint à l'équipe au moment de la transformation de la revue en périodique scientifique. Le travail et le dévouement de Mathieu, dans tous les aspects de production de Téoros, ont été remarquables.

Au nom du comité de direction et de rédaction, et en mon nom personnel, je souhaite saluer Mathieu, et le remercier vivement pour sa contribution marquante à la revue Téoros.

Alain A. GRENIER, Ph.D.

Directeur et rédacteur en chef 